\title{
La comunicación kinésica en árabe y su reconocimiento en el aprendizaje de la lengua árabe
}

\author{
Patricia Vela Moreno. Universidad de Granada \\ Bachir Mahyub Rayaa. Universidad de Granada
}

Recepción: 27.06.2018 | Aceptado: 2.10.2018

Correspondencia a través de ORCID: Bachir Mahyub Rayaa

iD 0000-0001-5400-5374

Citar: Vela Moreno, P. y Mahyub Rayaa, B. (2018). La comunicación kinésica en árabe y su reconocimiento en el aprendizaje de la lengua árabe. ReiDoCrea, 7, 348-362.

\begin{abstract}
Resumen: Este trabajo se centra en la comunicación no verbal, más en concreto, en el lenguaje corporal de oradores nativos de lengua árabe. En él pretendemos analizar el uso que hacen dos oradores árabes de la comunicación kinésica para, a partir de los resultados obtenidos, poder dar respuesta a una serie de interrogantes y objetivos marcados. Estos objetivos son, en primer lugar, averiguar si los participantes en el estudio son capaces de identificar o reconocer ciertos rasgos comunes del lenguaje no verbal en árabe (énfasis/refuerzo y negación). En segundo lugar, averiguar si conocen el significado de los mismos y, en su caso, saber si es gracias a que los han estudiado o por influencia de su entorno social, cultural, etc. Y por último, averiguar si el reconocimiento del significado de estos elementos del lenguaje no verbal va ligado o no al nivel de competencia lingüística en árabe y/o al contacto del encuestado con esta sociedad y su cultura. Método: Realizamos un estudio empírico en el cual, a través de dos encuestas realizadas a dos grupos de alumnos de Traducción e Interpretación, especialidad árabe, y de niveles distintos (inicial y avanzado), hemos obtenido una serie de datos que posteriormente se han analizado y discutido. De ello se coligen varias conclusiones relevantes y se averigua en qué medida se han alcanzado los objetivos iniciales. Resultados: El análisis gráfico de los datos nos indica que no existe una constante bien definida. Los resultados son marcadamente heterogéneos, si bien se aprecian tendencias llamativas. Conclusiones: Factores a priori determinantes como la procedencia de los sujetos -árabe o española-, el contacto con la cultura árabe, así como el nivel de competencia lingüística en árabe, no se han revelado determinantes en cuanto a la identificación de los gestos y el reconocimiento de su significado.
\end{abstract}

Palabras clave: Comunicación no verbal | Lengua árabe | Lenguaje corporal | Oradores árabes | Kinésica

\section{Arab Kinesics and its perception by Arabic learners}

\begin{abstract}
: this work focuses on non-verbal communication, more specifically on the body language of Arabic native speakers. We analyse in this paper the non-verbal communication and the body language of two Arab speakers in order to respond a series of questions and objectives initially marked. Our main objectives are: (i) to find out whether the respondents are able to identify and recognize certain features (emphasise and negation) of Arabic non-verbal language; (ii) to find out if they know the meaning of those features and, in that case, whether it is due to they having studied them or because of their social and cultural environment; (iii) and to find out whether the recognition of Arabic non-verbal language is linked to the level of linguistic competence in Arabic and/or to the contact the respondents have with Arab culture. Method: An empirical study based on two surveys was conducted. We surveyed two groups -initial and advanced- of Translation and Interpretation studies with Arabic as a first foreign language. The data we obtained were subsequently analysed to reach certain conclusions and find out the proposed objectives. Results: The graphical analysis shows non-significant differences. The results obtained are somewhat heterogeneous. Conclusions: Factors such as the respondents background, either Arabic or Spanish, the contact with the Arab culture, as well as the level of linguistic competence in Arabic, have not been decisive nor in terms of the identification of the gestures per se nor in their acknowledgement.
\end{abstract}

Keywords: Nonverbal communication | Arabic | Body language | Arab speakers | Kinesics

\section{Introducción}

El estudio de la comunicación no verbal en seres humanos, también llamada lenguaje no verbal, lenguaje corporal o comportamiento no verbal (non verbal behaviour), goza a día de hoy de una dilatada trayectoria. En los años 20 y 30 de siglo XX, la investigación comenzó a interesarse por la expresión facial y cómo esta faceta de la 
comunicación no verbal revelaba ciertos estados emocionales. Años más tarde se ponía el foco en la relación de determinadas manifestaciones del lenguaje corporal con la detección de trastornos neuronales. Entrada la década de los 50, investigadores estadounidense como Hall (1959) y Birdwhistell (1970) comenzaron a interesarse por la relación del lenguaje corporal con la cultura y el nivel educativo (Safadi y Valentine, 1990: 4). Con esta nueva tendencia aparecerían numerosos estudios comparativos entre culturas diferentes, así como entre seres humanos y animales. Los resultados de parte de estas investigaciones comenzaron a aplicase en el terreno de la formación intercultural en sectores como los negocios y el comercio internacional (cf. Landis y Brislin, 1983). En lo que respecta a la incidencia del lenguaje no verbal (LNV) en el acto comunicativo, aparecieron por entonces varias opiniones. Birdwhistell (1970) y Aylesworth (1979) consideran que el LNV que emitimos los humanos puede transmitir hasta un $65 \%$ del mensaje que queremos hacer llegar por la vía oral. Otros autores como Hall (1984) van incluso más allá al afirmar que el porcentaje que se transmite por medio del LNV podría alcanzar hasta un $90 \%$. Más allá de esta notoria variación de porcentajes, todos sugieren que una carga importante del mensaje que se pretende transmitir se puede deducir por medio del comportamiento no verbal del emisor. En nuestra opinión, aparte del enfoque observacional, seguimos necesitando más investigación empírica con el fin de validar o rechazar esta hipótesis.

Lo cierto es que, fruto de sus innumerables aplicaciones, la comunicación no verbal se ha convertido hoy día en un área interdisciplinar que abarca prácticamente todos los ámbitos de la vida en los que dos o más personas entran en contacto. La literatura previa distingue entre tres grandes tipos de comunicación no verbal: la paralingüística, aquella referida a los elementos no verbales resultantes de los parámetros de la voz (Poyatos, 2002: 3-20 y autores citados por éste); la kinésica, referida a los gestos y al LNV consciente e inconsciente (Birdwhistell, 1970; Poyatos, 2002: 185-186); y la proxémica, que se centra en la distribución del espacio personal y social durante la comunicación (Schmidt, 2013: 88-91).

En este trabajo nos centraremos en la vertiente kinésica, concretamente en el LNV que indica énfasis/refuerzo y negación entre oradores árabes y cómo es asimilado por estudiantes de lengua árabe del área de la traducción y la interpretación. El principal motivo que nos ha impulsado a realizar un estudio sobre la kinésica en la lengua y la cultura árabes es la escasez de trabajos empíricos previos sobre este tema en su relación con la enseñanza del árabe como lengua extranjera y la interpretación árabeespañol. De hecho, durante el proceso de recopilación bibliográfica y tras una larga búsqueda infructuosa, llegamos a la conclusión de que para aproximarnos a este tema estamos obligados a recurrir a obras que, sin llegar a abordar la comunicación no verbal en el binomio árabe-español, sí lo hacían en combinaciones lingüísticas como árabe-inglés. Esto nos proporcionaba al menos un marco teórico general acerca del LNV en los estudios interculturales y nos ofrecía ejemplos en los que se comparaba el LNV árabe con el estadounidense (Safadi y Valentine, 1990: 4). Del mismo modo, tuvimos que recurrir a trabajos sobre la comunicación no verbal en el marco de la enseñanza-aprendizaje del español como lengua extranjera (Monterubbianesi, 2011, Schmidt, 2013, entre otros).

Nuestro interés en la kinésica radica en la clave importancia que esta desempeña en todo acto comunicativo. Tal es su relevancia que existen situaciones que bien no se entenderían bien podrían derivar en un mal entendido si no se comprende el LNV implícito en ellas (Hall, 1984 y Poyatos, 1994). El hecho de que la comunicación kinésica podría variar en mayor o menor grado dependiendo de cada lengua y su cultura, y que los gestos y movimientos casi siempre contienen una cargar semántica, sea para apoyar el lenguaje verbal, o para suplirlo, hace que cobre un interés especial en la enseñanza-aprendizaje de las lenguas extranjeras. Este interés aumenta 
sustancialmente en el caso de lenguas asimétricas lingüística y culturalmente al idioma del aprendiz, como es el caso del árabe para los hablantes nativos de español y, dada la gran extensión del mundo árabe, para los propios árabes entre sí dependiendo de su país y zona de origen. Para un aprendiz de la lengua árabe basta con sumergirse en un entorno sociolingüístico árabe para descubrir la importancia que en ella tiene el LNV y advertir las distintas variaciones con respecto a su propia lengua y cultura.

Si bien carecemos hoy en día de una base empírica que verse sobre la inclusión del LNV en la enseñanza del árabe como lengua extranjera, lo cierto es que existe una cantidad nada desdeñable de prosa sobre el estudio de los gestos en árabe y sus significados (Feghali, 1997: 363-364). Los estudiosos de la sociolingüística, el orientalismo y la antropología humana -principalmente de EE. UU.- comenzaron a mostrar su interés por las tres facetas de la comunicación no verbal en la lengua y la cultura árabes en las últimas décadas del siglo pasado. En el trabajo mencionado más arriba de Safadi y Valentine (1990), titulado "Contrastive analyses of American and Arab nonverbal and paralinguistic communication", encontramos el primer estudio relativamente extenso sobre los gestos empleados por los hablantes árabes. Las autoras aplican en este trabajo modelos de comportamiento no verbal propuestos por lingüistas consolidados como Hall (1966), Birdwhistell (1970) o Sparhawk (1981) para comparar los gestos recopilados con el lenguaje gestual estadounidense. Estas autoras, sin llegar a conclusiones firmes, ofrecen un corpus de gestos realizados con diferentes partes del cuerpo, junto con sus significados en EE. UU. y en el mundo árabe. Es llamativo ver que, si bien algunos coinciden, existen muchos gestos sin equivalente y otros con significados diferentes.

No cabe duda de que en el comportamiento no verbal inciden variables personales y otras colectivas, como tampoco cabe duda de que buena parte de dicho comportamiento se transmite de generación en generación dentro de una misma sociedad dando lugar a un código consensuado y reconocido por todos sus miembros (cf. Hall, 1966). En lo que respecta la idiosincrasia árabe, si bien no aportan evidencias fundadas de cómo se obtuvo el dato ni dónde se puede localizar esta extensa lista, Samovar y Porter (1991) mencionan la conclusión alcanzada por un arabista norteamericano que afirma haber recopilado unos 247 gestos diferentes empleados en el LNV árabe como apoyo a la comunicación verbal. A este dato aproximado, habría que añadir la existencia de una variación en los gestos utilizados en todo el mundo árabe. Safadi Valentine (1990: 278) describen esta peculiaridad como "subtle physical differences that amount to great semantic variations".

Creemos, en definitiva, que la comunicación no verbal sigue siendo una asignatura pendiente en la enseñanza de la lengua árabe, así como en la traducción y la interpretación árabe-español. Prueba de ello es que en el proceso de recopilación bibliográfica no hemos hallado literatura previa al respecto. Desde nuestra experiencia en la formación de traductores e intérpretes de árabe se aprecia una gran laguna en este campo. Tampoco hemos hallado evidencias que se imparta enseñanza específica de este ámbito en todo el currículo de Traducción e Interpretación, especialidad árabe. Todo indica, por lo tanto, que se obvia su utilidad para anticipar el significado, deducir las partes del mensaje que se desconocen, negociar el sentido del mensaje, entre otras utilidades.

\section{Hipótesis y objetivos}

En el presente trabajo partimos de la hipótesis de que el dominio del LNV de oradores árabes -entendido como gestos y expresión facial y corporal- ayuda a deducir el significado de un mensaje oral en árabe que no se comprende o no se oye. 
Delimitando el marco de acción a los estudiantes de traducción e interpretación con árabe como primera lengua extranjera, nos gustaría asimismo saber si estos son capaces de identificar o reconocer ciertos rasgos del lenguaje no verbal en árabe; averiguar si conocen el significado de dichos rasgos y, en su caso, saber si su entorno sociocultural influye en que conozcan dichos gestos -como ya se ha mencionado, sabemos de antemano que estos estudiantes no han recibido formación previa en materia de comunicación no verbal en árabe. Por último, averiguar si el reconocimiento del significado de los rasgos del lenguaje no verbal va ligado o no al nivel de competencia lingüística en árabe y/o al contacto del encuestado con dicha cultura.

\section{Métodos}

Para realizar este estudio, tuvimos que buscar muestras reales de lenguaje no verbal en árabe en formato audiovisual. Esta tarea no ha sido nada sencilla. El primer obstáculo al que nos enfrentamos fue la imposibilidad de discriminar mediante palabras de búsqueda los ejemplos reales de LNV de otros vídeos que versan sobre este tema o sobre coaching pero desde un enfoque teórico-didáctico, por lo que eran inservibles para nuestro propósito. De este modo, el proceso de búsqueda duró mucho más tiempo del que nos habíamos planteado desde el inicio. Las muestras obtenidas pasaron por un proceso de criba en el que nos queríamos asegurar de que cumplieran una serie de parámetros. Ante la imposibilidad de encontrar vídeos que mostraran oradores de cuerpo completo -situación ideal para este estudio- tuvimos que conformarnos con que al orador se le enfocara de medio cuerpo (tronco superior), así como que se apreciasen con claridad sus gestos. Finalmente escogimos dos vídeos, uno de la región del Golfo y otro de Marruecos. La elección de dos regiones del mundo árabe tan alejadas fue deliberada. Queríamos saber si entre los sujetos encuestados había diferencias en el reconocimiento del LNV de cada uno de los oradores.

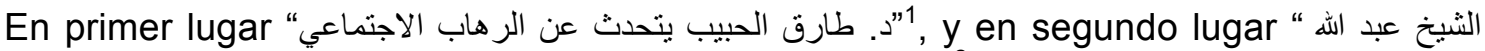
2. El orador del primer vídeo es un psicólogo nacido en Arabia Saudí que habla de la fobia social. El segundo vídeo es de un predicador marroquí al que se plantean consultas de carácter religioso. En este caso la consulta es sobre el adulterio cometido por el hombre debido a que su esposa no quiere cohabitar con él.

Tras la selección, elaboramos dos encuestas, una para cada vídeo ${ }^{3}$. Ambas están compuestas por dos secciones: una relativa al perfil del encuestado y otra sobre los vídeos que se visionarán. En el primer apartado se incorporan preguntas sobre los datos personales y el perfil lingüístico del sujeto que la cumplimenta, así como sobre su contacto con la cultura y sociedades árabes. A continuación, el segundo apartado se subdivide en otros dos (vídeo 1 y vídeo 2) y en ellos se pregunta por el orador y el tema de cada vídeo. Así mismo, se incluyen veinte preguntas en cada apartado sobre una serie de gestos realizados por el orador para constatar si el encuestado es capaz de reconocerlos y conoce su significado. Como se puede apreciar, cada una de las preguntas viene apoyada por uno o varios fotogramas, para que sea más sencilla la identificación del gesto. Cabe mencionar que en ciertos casos en los que la imagen no era clara, optamos por añadir una pequeña explicación verbal sobre los movimientos realizados por el orador.

Las encuestas fueron suministradas a alumnos de la Facultad de Traducción e Interpretación de la Universidad de Granada (especialidad árabe). Escogimos dos grupos, uno de nivel inicial y otro avanzado con el fin de poder obtener una visión

\footnotetext{
${ }^{1}$ Disponible en: https://goo.gl/uYehmD

${ }^{2}$ Disponible en: https://goo.g1/SRDeA3

${ }^{3}$ Disponible en: https://goo.gl/7sbRsP DOI: 10.6084/m9.figshare.7093172
} 
general del conocimiento que poseen acerca del LNV en árabe. El primer grupo (nivel avanzado) está formado por diez alumnos, mientras que el segundo (nivel inicial), lo componen siete. El cómputo global de participantes es por lo tanto de 17 alumnos.

Antes de comenzar las encuestas, brindamos a los participantes una pequeña presentación sobre el trabajo, los objetivos y pasos a seguir para cumplimentar la encuesta. Se les dijo que iban a visionar dos vídeos en los cuales hablaba un orador de origen árabe y que estos serían proyectados sin audio, para posteriormente contestar a una serie de preguntas sobre los gestos que realizaba cada uno. Se les pidió la máxima honestidad y se les recordó que la encuesta era totalmente anónima.

En el proceso de visionado se eliminó el sonido de los dos vídeos para que no hubiera ningún tipo de interferencia. Después de cada vídeo se suministró la encuesta correspondiente.

Tras finalizar las encuestas, se volcaron todos los datos obtenidos a un documento Excel para su análisis estadístico

\section{Resultados}

Como hemos indicado más arriban, presente estudio se centrará específicamente en los gestos de "énfasis/refuerzo" y de negación.
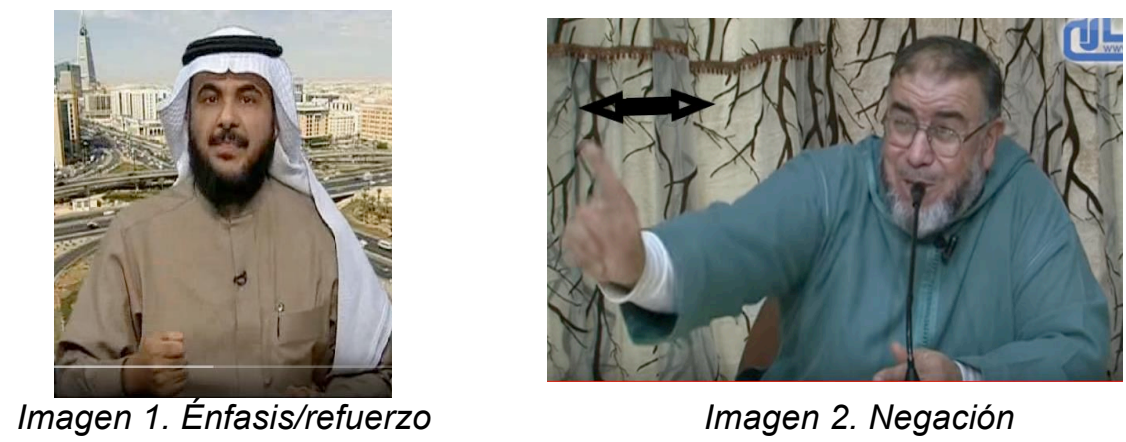

No obstante, en los vídeos proyectados había varios tipos de gestos presentes. Algunos de estos gestos son más comunes y fáciles de identificar que otros, puesto que son iguales que en español, universales o tienen el mismo significado en varias culturas. Dejaremos para la etapa de investigación futura el análisis del resto LNV detectado en los dos vídeos.

Empezaremos por la exposición y discusión de los resultados del primer bloque (énfasis/refuerzo). En este grupo se incluyen cuatro preguntas del vídeo $1\left(n^{\circ} 2,7,8\right.$ y 14) y otras seis del vídeo $2\left(n^{\circ} 1,2,7,8,13\right.$ y 17). Las capturas de todas ellas se pueden consultar en el enlace indicado en el apartado superior.

En primer lugar, expondremos los gráficos obtenidos a partir de los resultados de cada una de las preguntas seleccionadas para posteriormente realizar su análisis conjunto. Debemos mencionar aquí que los datos obtenidos de los dos grupos de encuestados (avanzado e inicial) se comentarán por separado, empezando por el grupo avanzado, ya que fue al primero al que se le hizo la encuesta.

En la codificación de los resultados emplearemos las siguientes abreviaturas:

1. S/A: el encuestado afirma saber el significado del gesto y además acierta.

2. S/NA: el encuestado afirma saber el significado del gesto, pero no acierta.

3. N/AP: el encuestado niega saber el significado del gesto pero acierta o se aproxima en la explicación aportada.

4. N/NA: el encuestado niega saber el significado del gesto y además no acierta ni se aproxima en su explicación. 
5. N/N: el encuestado niega saber el significado del gesto y tampoco aporta una explicación sobre qué cree que significa.

\section{Grupo de nivel avanzado. Bloque "gestos de énfasis/refuerzo"}

\section{Vídeo 1}

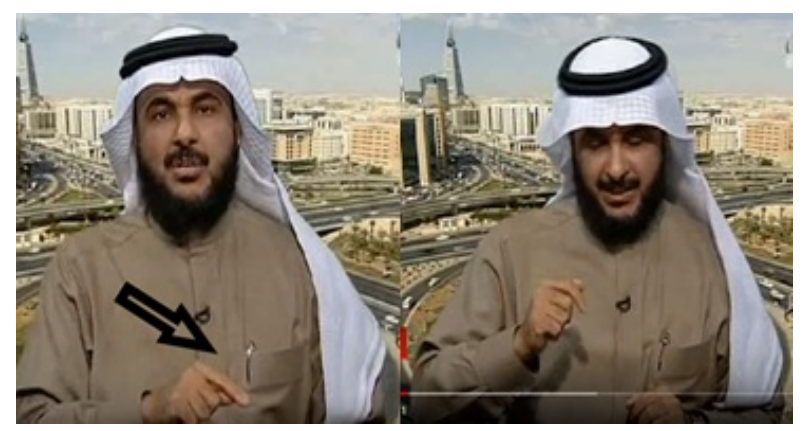

Imagen 3. Ejemplo de énfasis/refuerzo del vídeo $1^{4}$.

En la siguiente tabla se presentan de un modo sintetizado todas las respuestas de este bloque:

\begin{tabular}{lllllllllll} 
& S1 & S2 & S3 & S4 & S5 & S6 & S7 & S8 & S9 & S10 \\
P2 & S/NA & S/NA & N/N & N/NA & N/N & S/NA & N/N & N/NA & N/N & S/NA \\
P7 & S/A & S/A & S/A & N/AP & N/AP & N/NA & N/AP & N/N & N/N & N/AP \\
P8 & S/NA & S/A & N/N & N/AP & N/N & S/NA & N/NA & N/AP & N/N & S/NA \\
P14 & N/NA & S/NA & N/N & N/NA & N/N & S/NA & N/NA & N/AP & N/N & N/NA \\
\hline \multicolumn{6}{c}{ Tabla 1. Bloque de énfasis/refuerzo (vídeo 1). }
\end{tabular}

De los resultados que se exhiben en la tabla se colige que prácticamente todos los sujetos no aciertan en reconocer el significado de los gestos de este bloque. De hecho, solamente el sujeto 2 logra acertar el $50 \%$ de ellos. No obstante, la mayoría propone una posibilidad pero solo un $50 \%$ de los casos se aproxima. Véase el gráfico a continuación:

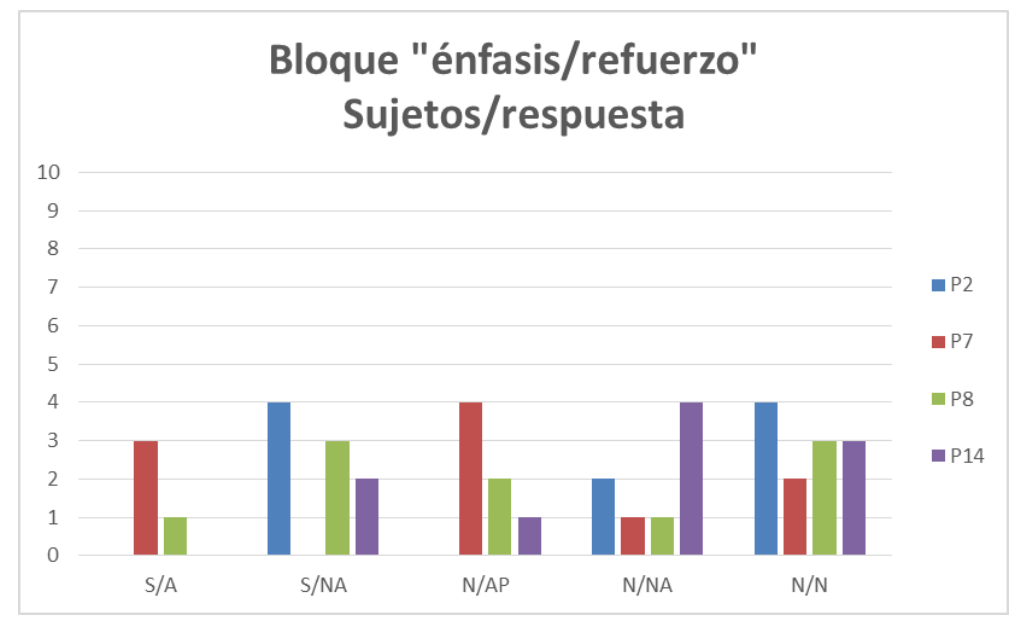

Gráfica 1. Respuestas de los sujetos en el bloque "énfasis/refuerzo" del vídeo 1.

\footnotetext{
${ }^{4}$ Para el resto de gestos, véase las preguntas 7 y 14 de la encuesta 1.
} 
Si cruzamos estos resultados con los perfiles de los encuestados (véase seguido tabla 2 ), observamos que el sujeto que más acierta es de nacionalidad marroquí, mientras que entre los que menos aciertan, predomina la nacionalidad española.

\begin{tabular}{|c|c|c|c|c|c|c|c|c|c|c|}
\hline & S1 & S2 & S3 & S4 & S5 & S6 & S7 & S8 & s9 & S10 \\
\hline Sexo & $M$ & $M$ & $\mathrm{M}$ & $M$ & $\mathrm{M}$ & $M$ & $M$ & $\mathrm{H}$ & $M$ & $\mathrm{H}$ \\
\hline Edad & 21 & 21 & 22 & 22 & 22 & 22 & 24 & 21 & 26 & 22 \\
\hline Nacionalidad & Esp & Marr & Esp & Esp & Esp & Marr & Esp & Esp & Esp & Esp \\
\hline Leng. materna & Esp & Bereber & $\begin{array}{l}\text { Esp- } \\
\text { IN }\end{array}$ & $\begin{array}{l}\text { Marr } \\
\text { - Esp }\end{array}$ & Sirio & Bereber & Esp & Esp & Esp & Esp \\
\hline L. extranj. 1 & $\bar{A} R$ & $\overline{A ́ R}$ & ÁR & ÁR & ÁR & ÁR & ÁR & ÁR & ÁR & ÁR \\
\hline L. extranj. 2 & IN & FR & PR & IN & IN & IN & IN & IN & IN & FR \\
\hline $\begin{array}{l}\text { Estudio desde } \\
\text { año } \mathrm{H} / \mathrm{mes}\end{array}$ & $\begin{array}{l}2014 / \\
61\end{array}$ & $\begin{array}{l}2014 / \\
60\end{array}$ & $\begin{array}{l}2013 \\
/ 70\end{array}$ & $\begin{array}{l}2014 \\
/ 60\end{array}$ & $\begin{array}{l}2014 / \\
60\end{array}$ & $\begin{array}{l}2014 / \\
100\end{array}$ & $\begin{array}{l}2011 / \\
36\end{array}$ & $\begin{array}{l}2014 / \\
35\end{array}$ & $2010 / 18$ & $\begin{array}{l}2014 / \\
100\end{array}$ \\
\hline Nivel considera & Med & $\begin{array}{l}\text { Avan } \\
z\end{array}$ & Med & $\begin{array}{l}\text { Avan } \\
\mathrm{z}\end{array}$ & Med & $\begin{array}{l}\text { Avan } \\
z\end{array}$ & Med & Med & Med & Med \\
\hline $\begin{array}{l}\text { Contacto } \\
\text { cultura AR }\end{array}$ & No & Siemp & No & Siemp & Siemp & No & $\begin{array}{l}9 \\
\text { mese } \\
\mathrm{s}\end{array}$ & $\begin{array}{l}10 \\
\text { mese } \\
\mathrm{s}\end{array}$ & No & No \\
\hline
\end{tabular}

Tabla 2. Perfil sociolingüístico del grupo avanzado.

\section{Vídeo 2}

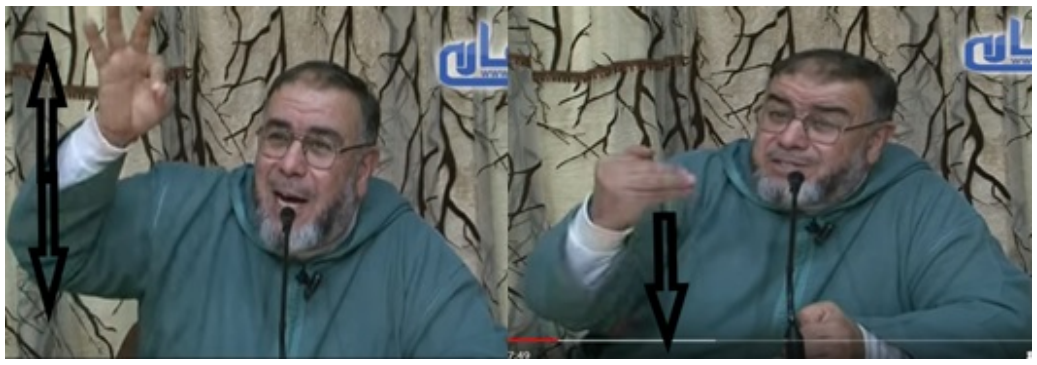

Imagen 4. Ejemplo de énfasis/ refuerzo del vídeo $2^{5}$.

En la siguiente tabla se presentan de un modo sintetizado todas las respuestas de este bloque:

\begin{tabular}{lllllllllll} 
& S1 & S2 & S3 & S4 & S5 & S6 & S7 & S8 & S9 & S10 \\
P1 & S/NA & S/NA & S/NA & N/NA & N/NA & S/NA & S/NA & N/N & N/N & N/NA \\
P2 & S/NA & N/NA & S/A & N/AP & N/AP & N/NA & N/AP & N/AP & N/N & N/AP \\
P7 & N/N & N/AP & S/NA & N/N & N/N & N/N & N/N & N/N & N/N & N/AP \\
P8 & N/N & S/NA & N/N & N/N & N/N & N/N & N/N & N/N & N/N & N/NA \\
P13 & S/NA & S/NA & S/NA & N/NA & S/NA & N/N & S/NA & N/NA & N/NA & N/NA \\
P17 & S/NA & S/A & S/NA & S/A & S/A & S/NA & S/A & N/AP & N/N & S/A \\
\hline \multicolumn{8}{c}{ Tabla 3. Bloque de énfasis/refuerzo (vídeo 2). }
\end{tabular}

De los resultados que se exhiben en la tabla se colige que casi todos los sujetos no aciertan en reconocer el significado de los gestos de este grupo. De hecho, solamente en torno al $16 \%$ de los casos, los sujetos son capaces de acertar o aproximarse en su significado. Véase el gráfico a continuación:

\footnotetext{
${ }^{5}$ Para el resto de gestos, véase preguntas $1,8,13$ y 17 de la encuesta 2.
} 


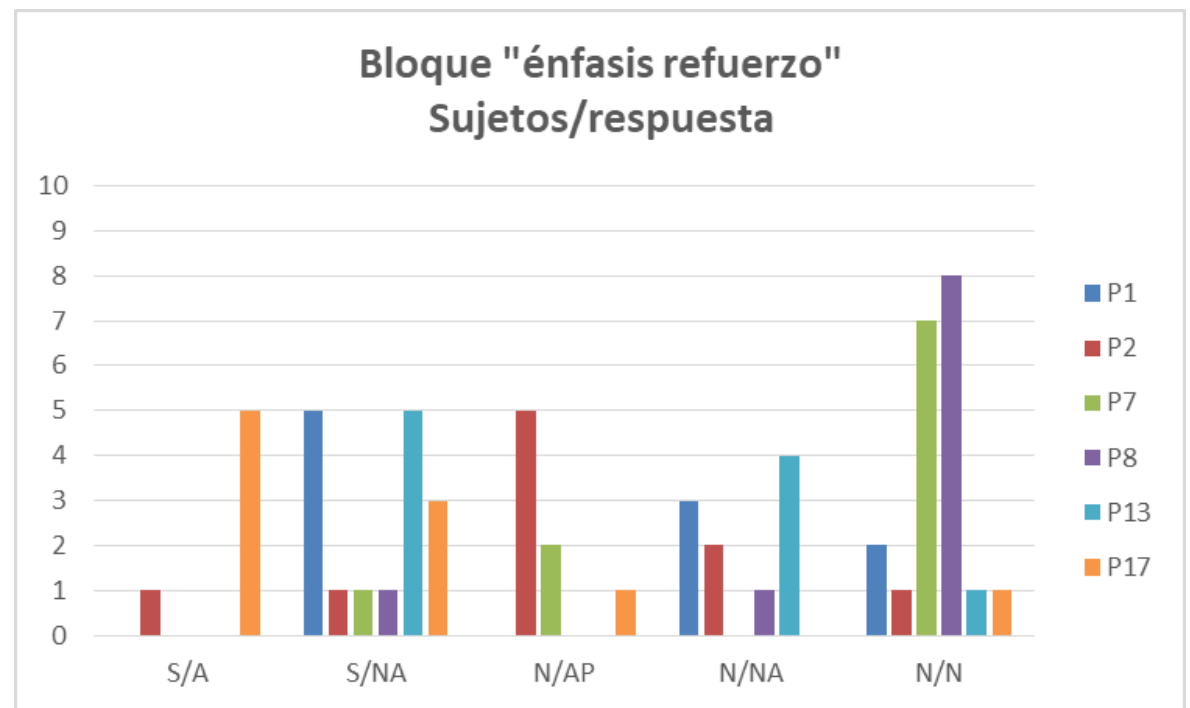

Gráfica 2. Respuestas del grupo avanzado al bloque "énfasis/refuerzo" (vídeo 2).

Si cruzamos estos resultados con los perfiles de los encuestados (véase tabla 2), observamos que entre los que más aciertan hay un equilibrio en cuanto a la nacionalidad de los sujetos y la mayoría de ellos ha estado en contacto con la cultura árabe.

Al igual que en el vídeo 1, si analizamos los datos del vídeo 2, podemos llegar a la conclusión de que la mayoría de los encuestados del grupo avanzado no es capaz de identificar ni conocer el significado de los gestos de énfasis y refuerzo, dejando ver que tanto la procedencia como el contacto con la cultura árabe no son factores determinantes a la hora de identificar los gestos de este bloque y sus respectivos significados.

\section{Grupo de nivel avanzado. Bloque “gestos de negación”. Vídeo 1}

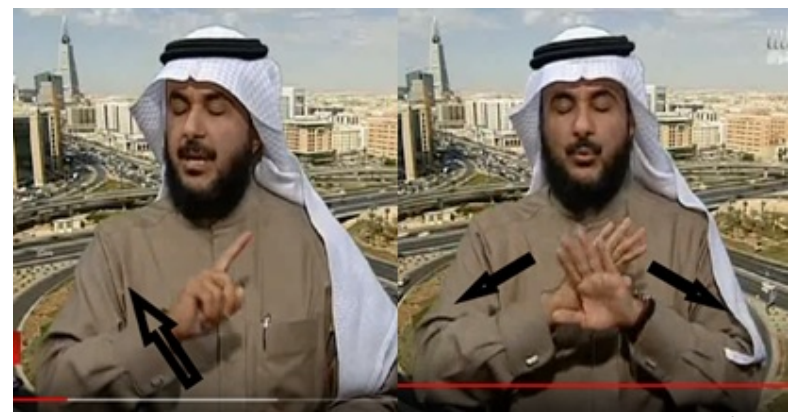

Imagen 5. Ejemplo de negación del vídeo $1^{6}$.

En la siguiente tabla se presentan de un modo sintetizado todas las respuestas de este bloque:

\begin{tabular}{lllllllllll} 
& S1 & S2 & S3 & S4 & S5 & S6 & S7 & S8 & S9 & S10 \\
P11 & N/AP & S/A & N/N & S/A & N/AP & S/A & N/AP & N/AP & N/AP & N/NA \\
P17 & N/N & S/A & N/AP & N/NA & N/N & S/A & N/AP & N/AP & N/N & S/A \\
P19 & S/A & N/AP & S/A & N/NA & N/NA & N/NA & N/NA & N/NA & N/N & S/A \\
\hline la 4. Respuestas del grupo avanzado en el bloque de negación (vídeo 1).
\end{tabular}

\footnotetext{
${ }^{6}$ Para el resto de gestos, véase pregunta 17 de la encuesta 1.
} 
De los resultados que se exhiben en la tabla 4 se colige que casi todos los sujetos niegan identificar los gestos de este grupo. Sin embargo, más de la mitad de los que aportan un posible significado, logra acertar o aproximarse. Véase el gráfico a continuación:

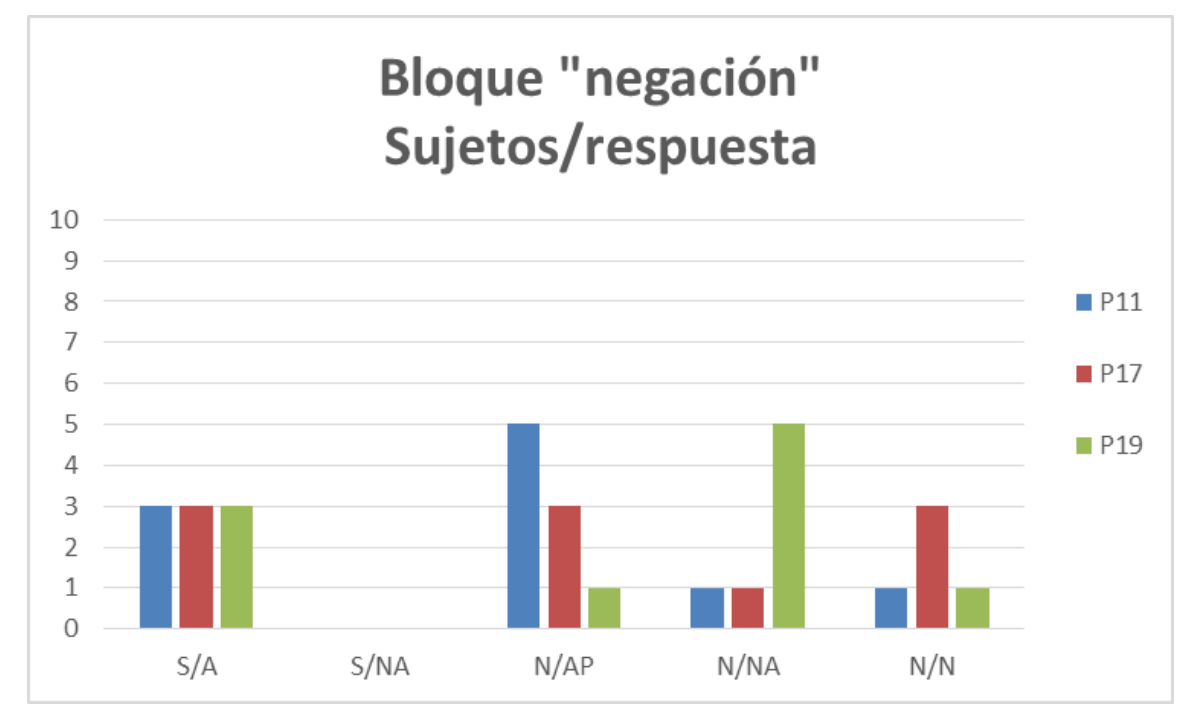

Gráfica 3. Respuestas del grupo avanzado al bloque "negación” (vídeo 1).

Si cruzamos estos resultados con los perfiles de los encuestados (véase tabla 2), observamos que entre los que más aciertan en este caso, predominan los de nacionalidad española.

\section{Vídeo 2}

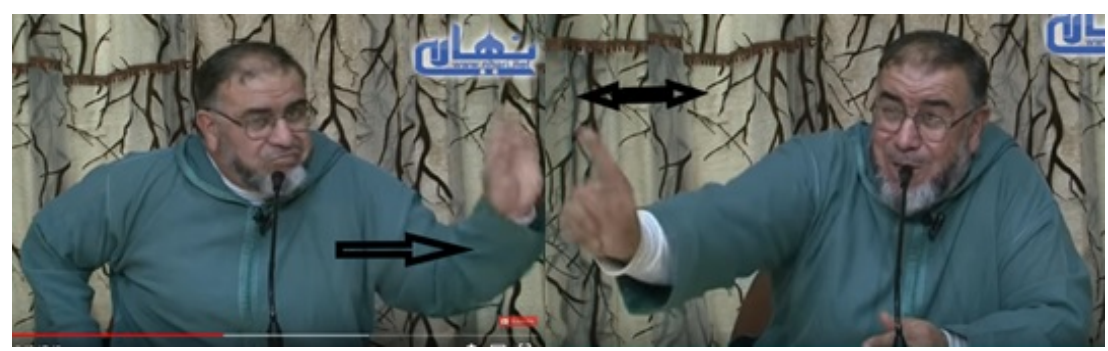

Imagen 6. Ejemplo de negación del vídeo 2.

En la siguiente tabla se presentan de un modo sintetizado todas las respuestas de este bloque:

\begin{tabular}{rcccccccccc} 
S1 & S2 & S3 & S4 & S5 & S6 & S7 & S8 & S9 & S10 \\
P14 & N/N & N/NA & N/N & N/N & N/N & N/N & N/N & N/NA & N/N & N/AP \\
P19 & S/A & S/A & N/N & S/A & N/N & N/AP & N/NA & N/N & N/N & N/AP \\
\hline Tabla 5. Respuestas del grupo avanzado en el bloque de negación (vídeo 2).
\end{tabular}

De los resultados que se exhiben en la tabla se colige que casi todos los sujetos no son capaces de reconocer los gestos de este grupo. De hecho, solamente en torno al $30 \%$ de los casos, los sujetos son capaces de acertar o aproximarse a su significado. Véase el gráfico a continuación: 


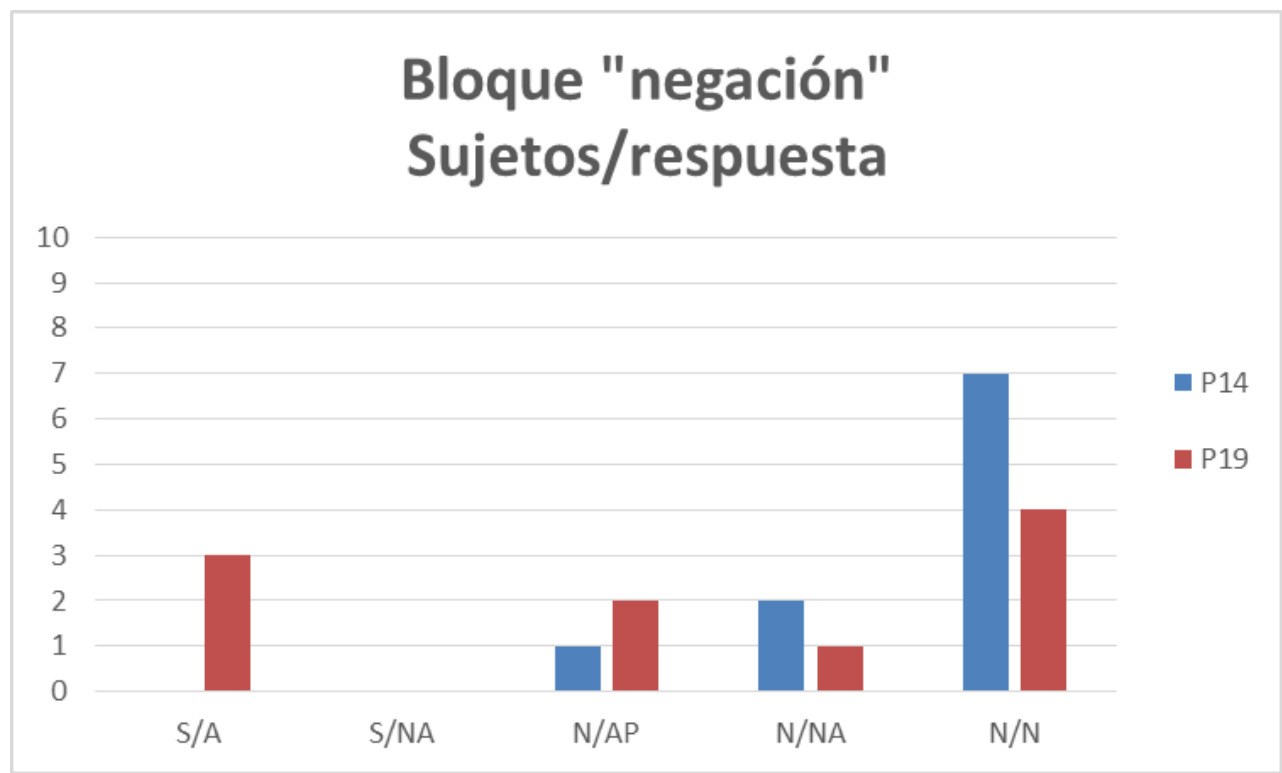

Gráfica 4. Respuestas del grupo avanzado al bloque "negación” (vídeo 2).

Si cruzamos estos resultados con los perfiles de los encuestados (véase tabla 2), observamos que entre los sujetos que aciertan a reconocer el gesto de la pregunta 19, destacan ligeramente los encuestados de origen árabe (S2, S4 y S6) y que afirman poseer un nivel avanzado de árabe. Se trata en los tres casos de personas de ascendencia marroquí. Sin embargo, vuelve a llamar la atención que los sujetos 1 y 10 , de origen español y sin estancias en entornos de cultura árabe, hayan acertado por encima de los demás. Mirando el perfil de estos sujetos, se aprecia que están entre el grupo que más horas de aprendizaje dedica a la lengua árabe.

Analizando los datos de los dos vídeos conjuntamente, podemos llegar a la conclusión de que la mayoría de encuestados no son capaces de identificar los gestos de negación. Sin embargo, en torno al $50 \%$ sí logra acertar o aproximarse a su significado. Dada la reducida muestra, no podemos saber a ciencia cierta qué factores intervienen en este caso. Entre los posibles, se podría apuntar a que los gestos de negación están más universalmente estandarizados, a la exposición a entornos de cultura árabe, procedencia del mismo país que el orador e incluso el número de horas que se dedican al aprendizaje de la lengua árabe.

\section{Grupo de nivel inicial. Bloque "Gestos de énfasis/refuerzo" \\ Vídeo 1}

En la siguiente tabla se presentan de un modo sintetizado todas las respuestas de este bloque:

$\begin{array}{llllllll} & \text { S1 } & \text { S2 } & \text { S3 } & \text { S4 } & \text { S5 } & \text { S6 } & \text { S7 } \\ \text { P2 } & \text { N/AP } & \text { N/N } & \text { N/AP } & \text { N/NA } & \text { N/NA } & \text { N/N } & \text { S/NA } \\ \text { P7 } & \text { N/AP } & \text { N/AP } & \text { N/NA } & \text { N/NA } & \text { N/NA } & \text { S/A } & \text { S/NA } \\ \text { P8 } & \text { N/NA } & \text { S/NA } & \text { S/NA } & \text { N/NA } & \text { N/NA } & \text { N/N } & \text { N/NA } \\ \text { P14 } & \text { N/NA } & \text { S/NA } & \text { S/NA } & \text { N/NA } & \text { N/NA } & \text { S/A } & \text { S/NA }\end{array}$

Tabla 6. Respuestas del grupo inicial al bloque "énfasis/refuerzo" (vídeo 1).

De los resultados que se exhiben en la tabla 6 se colige que casi todos los sujetos no son capaces de reconocer los gestos de este grupo. De hecho, solamente en aproximadamente el $21 \%$ de los casos los sujetos son capaces de acertar o aproximarse a su significado. Véase el gráfico a continuación: 


\section{Bloque "énfasis/refuerzo" Sujetos/respuesta}

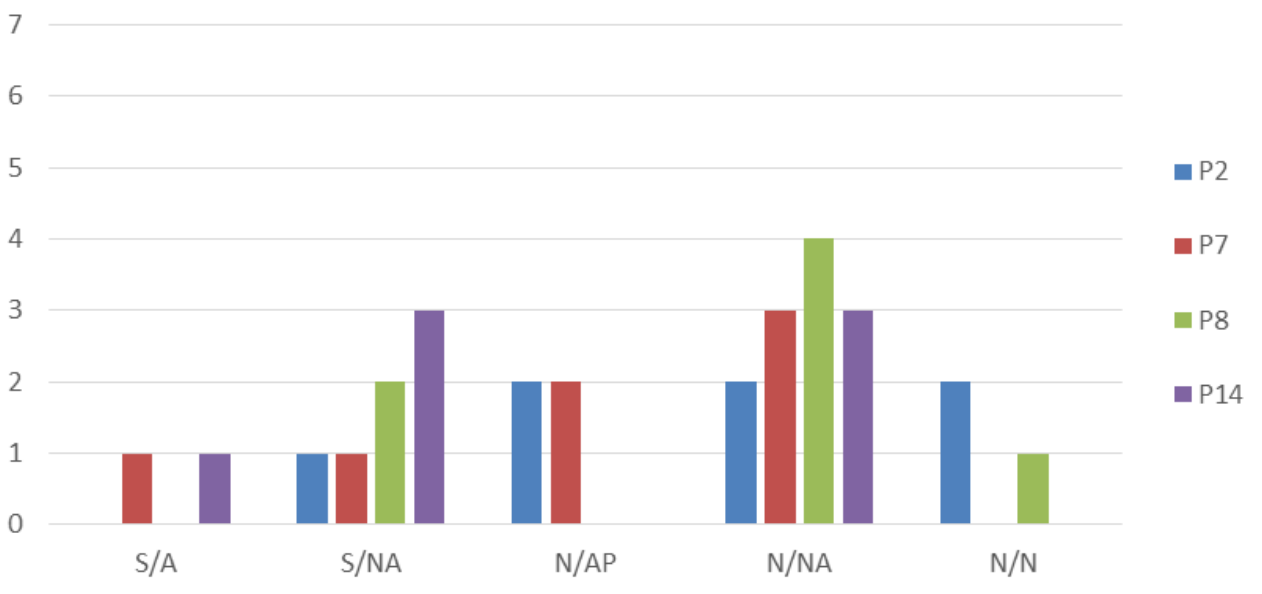

Gráfica 5. Respuestas del grupo inicial al bloque "énfasis/refuerzo" (vídeo 1).

Si cruzamos estos resultados con los perfiles de los encuestados (véase seguido tabla 7), observamos que entre los que más aciertan, predominan los de nacionalidad española y se trata de los sujetos con dicha nacionalidad que más horas han dedicado al aprendizaje de la lengua árabe.

\begin{tabular}{|c|c|c|c|c|c|c|c|}
\hline & S1 & S2 & S3 & S4 & S5 & S6 & S7 \\
\hline Sexo & $\mathrm{H}$ & $\mathrm{M}$ & $\mathrm{M}$ & $\mathrm{M}$ & $\mathrm{M}$ & $\mathrm{M}$ & $\mathrm{H}$ \\
\hline Edad & 20 & 20 & 21 & 19 & 21 & 19 & 22 \\
\hline Nacionalidad & Esp & Esp & Túnez & Esp & Túnez & Esp & Egipto \\
\hline L. Materna & Esp & Esp & DialecTN & Esp & DialecTN & Esp & DialecTN \\
\hline L. $B$ & Árabe & Árabe & $\mathrm{Fr} / \mathrm{Esp}$ & Árabe & Francés & Árabe & Español \\
\hline L. C & IN/RUM & ALE & $\mathrm{IN}$ & $\mathrm{IN}$ & ESP & RUS & IN \\
\hline Estudio desde año $\mathrm{H} / \mathrm{mes}$ & $\begin{array}{l}2015 / \\
50 \\
\end{array}$ & $\begin{array}{l}2015 / \\
14\end{array}$ & Siemp & $\begin{array}{l}2015 / \\
16 \\
\end{array}$ & Primaria/8 & $\begin{array}{l}2016 / \\
150 \\
\end{array}$ & Siemp \\
\hline Nivel considera & Bajo & Med & Avanz & Med & Bajo & Med & $\mathrm{NS} / \mathrm{NC}$ \\
\hline Contacto cultura AR & No & Amigo & Siempre & Viaje & No & 1 año & Siempre \\
\hline
\end{tabular}

Tabla 7. Perfil sociolingüístico de los encuestados del grupo inicial.

\section{Vídeo 2}

En la siguiente tabla se presentan de un modo sintetizado todas las respuestas de este bloque:

$\begin{array}{lllllll}\text { S1 } & \text { S2 } & \text { S3 } & \text { S4 } & \text { S5 } & \text { S6 } & \text { S7 }\end{array}$

P1 N/NA S/NA S/NA N/NA N/NA S/NA N/NA

$P 2 \quad N / N \quad S / N A \quad N / N \quad N / N A \quad N / N A \quad N / N A \quad S / A$

$\begin{array}{lllllll}P 7 & N / N & N & N & N & N & N\end{array} / N A \quad N / N A \quad S / N A \quad N / N A$

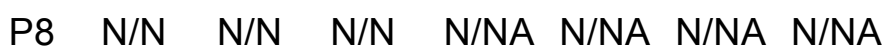

P13 N/NA S/NA N/N N/NA N/NA S/NA S/NA

P17 N/AP S/NA S/A N/AP N/NA N/AP S/A

Tabla 8. Respuestas del grupo inicial al bloque "énfasis/refuerzo" (vídeo 2). 
De los resultados que se exhiben en la tabla se colige que la mayoría de los sujetos no son capaces de identificar los gestos de este grupo. De hecho, solamente en torno al $14 \%$ de los casos, los sujetos son capaces de acertar o aproximarse en su significado. Véase el gráfico a continuación:

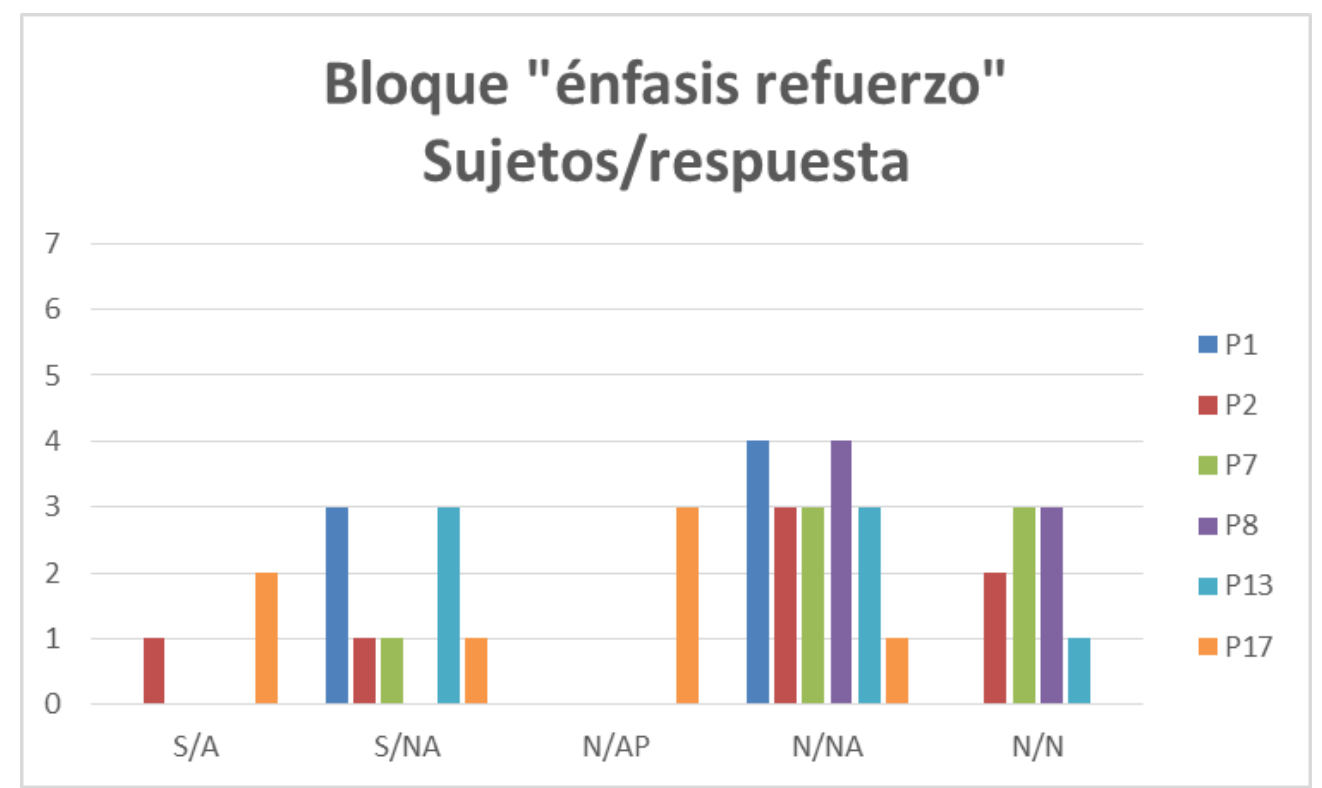

Gráfica 6. Respuestas del grupo inicial al bloque "énfasis/refuerzo" (vídeo 2).

Si cruzamos estos resultados con los perfiles de los encuestados (véase tabla 7), observamos que el sujeto que más acierta (S7) es de nacionalidad árabe. Entre el resto de sujetos que logran aproximarse al significado de alguno de los gestos, predomina los que son de nacionalidad española y han dedicado más tiempo al aprendizaje de la lengua árabe.

Si analizamos los datos de los dos vídeos conjuntamente, llegamos a la conclusión de que, al igual que los encuestados del grupo de nivel avanzado, la mayoría de sujetos del grupo inicial no son capaces de identificar ni conocer el significado de los gestos de énfasis y refuerzo en lengua árabe.

\section{Grupo de nivel inicial. Bloque "gestos de negación”}

\section{Vídeo 1}

En la siguiente tabla se presentan de un modo sintetizado todas las respuestas de este bloque:

\begin{tabular}{llllllll} 
& S1 & S2 & S3 & S4 & S5 & S6 & S7 \\
P11 & N/AP & S/NA & S/A & N/AP & S/A & S/A & S/A \\
P17 & N/NA & S/NA & N/N & N/AP & N/NA & S/NA & S/A \\
P19 & N/AP & S/NA & N/N & S/A & N/AP & S/NA & S/A \\
\hline
\end{tabular}

Tabla 9. Respuestas del grupo inicial al bloque "negación" (vídeo 1).

De los resultados que se exhiben en la tabla 9 se colige que el $50 \%$ de los sujetos afirma reconocer los gestos de este bloque, aunque solo en la mitad de los casos logran acertar su significado. Con respecto al $50 \%$ que no logra identificar los gestos, ligeramente en más de la mitad de los casos, los encuestados se aproximan a su significado. Véase el gráfico a continuación: 


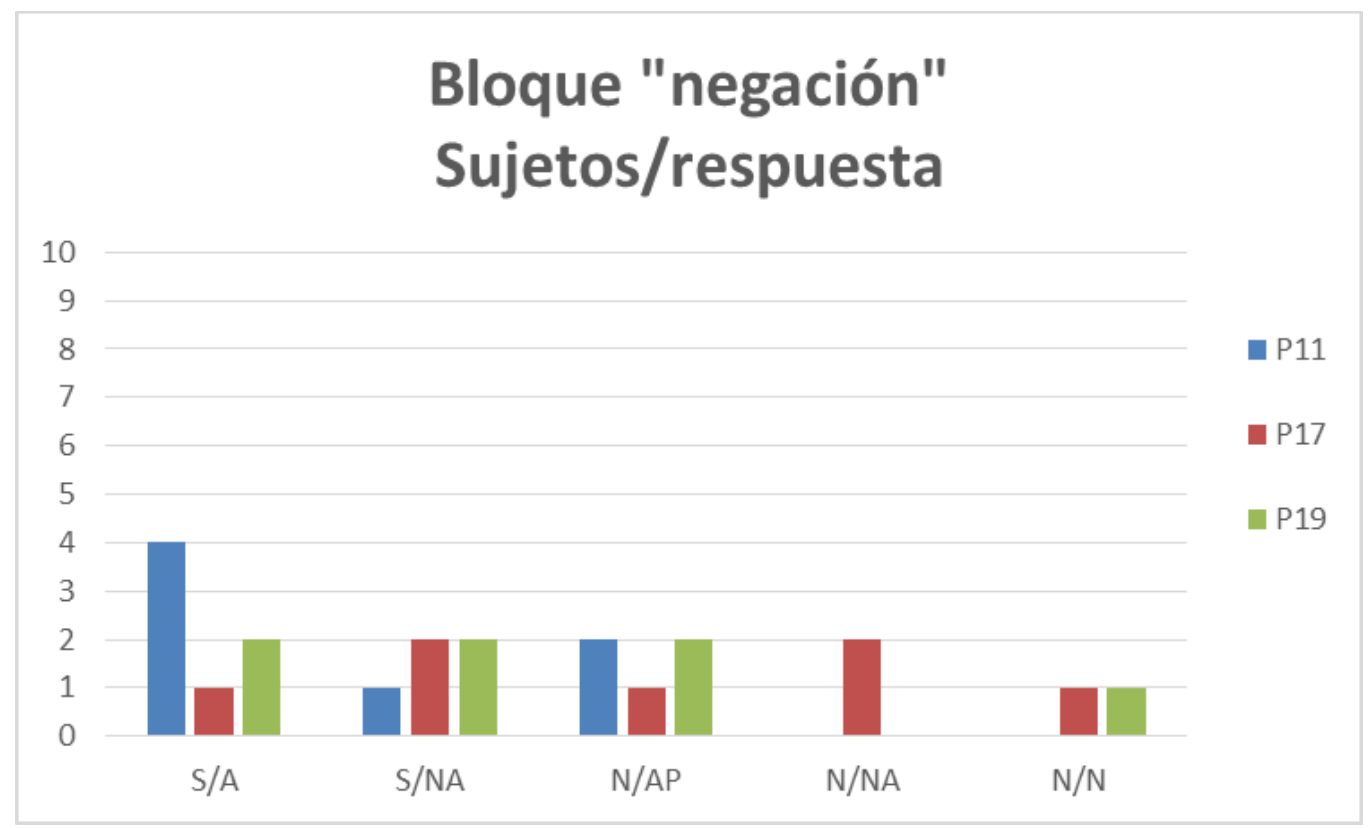

Gráfica 7. Respuestas del grupo inicial al bloque "negación" (vídeo 1).

Si cruzamos estos resultados con los perfiles de los encuestados (véase tabla 7), observamos que el sujeto $S 7$ vuelve a ser el que más acierta, concretamente acierta el $100 \%$ de los casos. Como vimos antes este participantes es de nacionalidad árabe. Entre el resto de sujetos que logran acertar o aproximarse al significado de alguno de los gestos, el porcentaje de árabes y españoles es el mismo.

\section{Vídeo 2}

En la siguiente tabla se presentan de un modo sintetizado todas las respuestas de este bloque:

\begin{tabular}{llllllll} 
& S1 & S2 & S3 & S4 & S4 & S5 & S6 \\
P14 & N/NA & S/NA & S/A & N/AP & N/N & N/NA & S/NA \\
P19 & N/AP & N/N & S/NA & N/AP & N/AP & S/A & S/A \\
\hline
\end{tabular}

Tabla 10. Respuestas del grupo inicial al bloque "negación" (vídeo 2).

De los resultados que se exhiben en la tabla 10 se colige que la mayoría de los encuestados no son capaces de identificar el gesto de la pregunta 14 ni de acertar o aproximarse a su significado. Por el contrario, en la pregunta 19, podemos observar que se invierten las tornas y ahora 5 de los 7 sujetos aciertan o se aproximan en su significado. Véase el gráfico a continuación: 


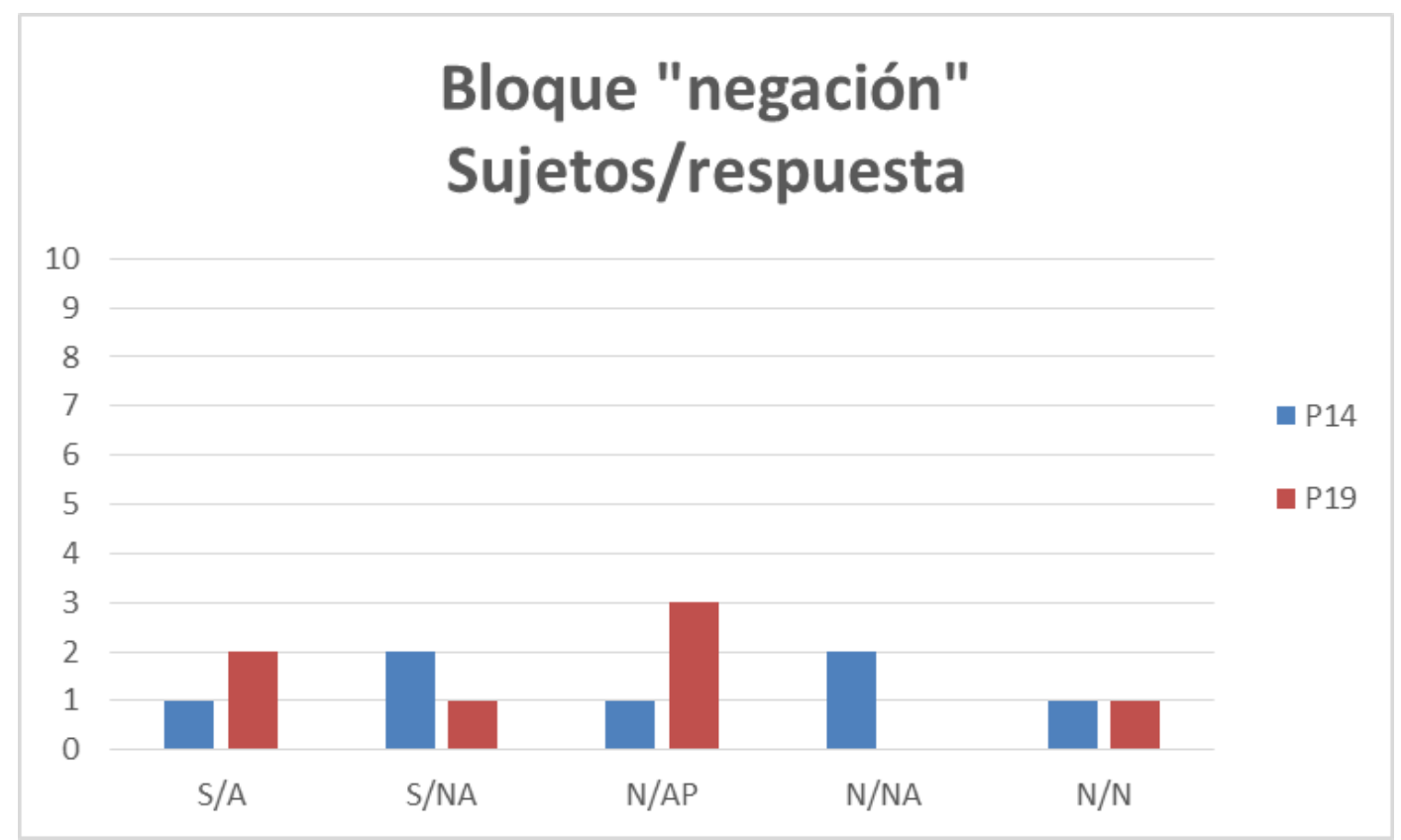

Gráfica 8. Respuestas del grupo inicial al bloque "negación” (vídeo 2).

Si cruzamos estos resultados con los perfiles de los encuestados (véase tabla 7), llama la atención que el sujeto 4 , aunque no identifica los gestos, se aproxima a su significado en todos los casos. Se trata de una persona con nacionalidad española y que ha estado muy poco en contacto con la cultura árabe.

Analizando los datos de los dos vídeos conjuntamente, podemos llegar a la conclusión de que alrededor del $50 \%$ de los encuestados de nivel inicial logra identificar la mayoría de gestos del bloque de negación, lo que supone un cambio visible con respecto al bloque anterior de énfasis/refuerzo. Por lo tanto, al igual que el grupo de nivel avanzado, este bloque resulta más sencillo de interpretar.

\section{Discusión}

Tras el análisis de los resultados obtenidos en los dos grupos de alumnos (avanzado e inicial), y teniendo en cuenta que la muestra de estudio es relativamente reducida, no podemos llegar a conclusiones firmes. Los resultados no indican una tendencia clara, si bien en algunos gestos sí se aprecia un denominador común entre los que aciertan y los que no. Para mayor seguridad, habría que realizar una segunda encuesta a una población mayor y compaginarla con una entrevista posterior en la que se les pregunte a los participantes acerca de los factores que le han llevado a tomar una respuesta $u$ otra. Solo así podremos determinar con mayor precisión los factores de incidencia reales.

Como se apunta más arriba, factores como la procedencia, ya sea árabe o española, el contacto con la cultura árabe, así como el nivel de competencia lingüística en esta lengua (árabe), no se han revelado determinantes o discriminatorios en cuanto a la identificación de los gestos y conocimiento de su significación en todos los casos. Solo en determinados rasgos estos tres factores parecen coincidir en los sujetos que aciertan.

A raíz de esto, la intuición de cada sujeto podría cobrar importancia como hemos podido apreciar en algunos casos. Esta faceta subjetiva y difícil de acotar se adquiere tanto en el entorno cultural como con el continuo aprendizaje de la lengua y la 
observación de sus hablantes. Precisamente aquí detectamos una verdadera laguna: cuando una persona se dispone a aprender una lengua, sea cual sea, se le da principalmente importancia a las cuestiones gramaticales y léxicas, dejando de lado los aspectos no verbales de la comunicación, que son igual de importantes que los verbales. Autores como Birdwhistell (1970) y Aylesworth (1979) consideran que el lenguaje no verbal que emitimos los humanos puede transmitir hasta un $65 \%$ del mensaje que queremos hacer llegar por la vía oral. Otros autores como Hall (1984) van incluso más allá al afirmar que el porcentaje que se transmite por medio del lenguaje no verbal podría alcanzar hasta un $90 \%$.

Esto se puede deber a, como mencionamos anteriormente, una falta de material didáctico y de énfasis en este tema; pero también, a que a lo largo del tiempo nos hemos ido centrando simplemente en los aspectos básicos de los códigos lingüísticos dejando los códigos no verbales y los aspectos pragmáticos de la comunicación relegados a un segundo plano. La competencia comunicativa no verbal se adquiere principalmente fuera del aula, pero dentro de ella se pueden enseñar los gestos más comunes, los bien vistos y los soeces, entre otros contenidos.

El presente estudio constituye una primera toma de contacto con este tema tan amplio y apasionante. Se hace necesario seguir investigando en el futuro para arrojar más luz sobre este campo en lengua árabe.

\section{Referencias}

Aylesworth, T. G. (1979). Understanding Body Talk. New York: Franklin Watts.

Birdwhistell, R. L. (1970). Kinesics and Context. Philadelphia: University of Pennsylvania Press.

Ekman, P. (1974). Darwin and Facial Expression. New York: Academic Press.

Ekman, P., Friesen, W. V. (1969). The repertoire of nonverbal behavior: Categories, origins, usage, and coding. Semiotica, 1(1), 49-98.

Escanciano, I. R. (2010). Lenguaje no verbal: Cómo gestionar una comunicación de éxito. La Coruña: Netbiblo.

Feghali, E. (1997). Arab cultural communication patterns. International Journal of Intercultural Relations, 21(3), 345-378.

Hall, E. T. (1959). The silent language. New York: Doubleday.

Hall, E. T. (1984). The dance of life: Another dimension of time. New York: Doubleday.

Monterubbianesi, M. G. (2011). La comunicación no verbal del español actual a partir de la filmografía de Pedro Almodóvar y su aplicación a la didáctica de E/LE (tesis doctoral). Universidad de Granada.
Pease, A. (2011). El lenguaje del cuerpo: cómo interpretar a los demás a través de sus gestos. Barcelona: Círculo de Lectores.

Poyatos, F. (2002). Nonverbal communication across disciplines (vol. 1, 2 y 3). Amsterdam: John Benjamins

Poyatos, F. (1994). La comunicación no verbal. Madrid: Istmo.

Safadi, M., \& Valentine, C. A. (1990). Contrastive analyses of American and Arab nonverbal and paralinguistic communication. Semiotica. 82 (3/4), 269-292.

Schmidt, S. (2013). Proxémica y comunicación intercultural: la comunicación no verbal en la enseñanza de E/LE (tesis doctoral). Universitat Autònoma de Barcelona.

Samovar, L. A. \& Porter, R. E. (1991). Communicution between cultures. California: Wadsworth.

Sparhawk, C. M. (1981). Contrastive identity features of Persian gesture. In A. Kendon (Ed.), Nonverbal communication, interaction and gesture (pp. 421-456). Berlin: Mouton.

Yule, G. (2007). El lenguaje. Madrid: Akal. 